\title{
Clinical-Pathological Features of an Occult Mixed Mucinous Male Breast Cancer: A Case Report
}

Ilaria Lamberti*, Adriana Bonifacino, Stefania Scarpino, Sandra Villani, Rita Mancini, Elisabetta Carico, Maria Rosaria Giovagnoli and Enrico Giarnieri

Department of Clinical and Molecular Medicine, Sapienza University of Rome, Rome, Italy

\begin{abstract}
Mucinous carcinoma of the male breast is an uncommon malignant breast neoplasm and its diagnoses remain difficult. It is probably due to such a low rate of breast cancer cases that men tend to be diagnosed at an older age than women and with a later stage of the disease. We describe a case of a 69-year-old male who displayed a palpable lump in his right axilla several years ago, showing signs of cutaneous adnexal mucinous adenocarcinoma after biopsy. After six years and several clinical examination and systemic investigation without results, the patient underwent to fine needle aspiration cytology and subsequently a biopsy of a mass with irregular margins in the retroareolar region of his right breast. The final diagnosis was of a mixed mucinous breast cancer with neuroendocrine differentiation. The tumor cells phenotype showed Synaptophisin (+), CEA (+/-), CK-20 (-), CK-7 $(+)$, TTF-1 (-), estrogen receptor (-), progesterone $(-)$ and HER $2(++)$. These results were unusual for a mucinous male breast carcinoma. In the presence of a lesion in the axillary area with no specific primary origin, breast cancer should never be ruled out, even in the absence of clinical evidence and with an immunohistochemical pattern not indicative of mammary origin.
\end{abstract}

Keywords: Male breast cancer; Mixed mucinous cancer; Occult primary neoplasms

\section{Introduction}

Male breast cancer (MBC) is infrequently observed in clinical practice and, therefore, unusual cases are not completely characterized and described. Male patients and physicians are less suspicious of such a disease and this may sometimes cause a diagnostic delay [1]. Only $48 \%$ of mucinous breast cancer is diagnosed at stage I/II of the disease so, in comparison with women, men tend to be diagnosed at a later stage of the disease. Therefore, its prognosis has not been significantly improved over the past 25 years [2]. Data from surveillance, epidemiology and end results (https://seer.cancer.gov) show that $93.7 \%$ of MBCs are ductal or unclassified carcinomas, and only $1.5 \%$ are lobular. Other tumor types include papillary (2.6\%) and mucinous (1.8\%) lesions [3]. Its incidence shows an increasing tendency and accounts for significant morbidity and mortality, showing a peak of incidence at around 60 years [4]. In addition to the better elucidation of the role of BRCA2, an increasing incidence of $\mathrm{MBC}$ has renewed interest in the pathogenesis and morphological features, clarifying specific differences between male and female breast cancer. Significant differences between male and female breast cancer have been reported. These regard the expression of a variety of biological parameters including hormone receptors such as estrogen and progesterone and c-erbB-2 receptors [5-7]. While male ductal intraepithelial neoplasia compared to similar lesions in females show a distinct histological profile, lesions are morphologically indistinguishable in invasive carcinomas [8,9]. There are 12 different types of breast cancer, of which infiltrating duct carcinoma is the most common. Mucinous carcinoma of the breast accounts for about $2 \%$ of breast carcinomas. Since male breast cancer is about 100 times less common than female breast cancer, mucinous carcinoma of the male breast is very rare [10]. It is probably due to such a low rate of breast cancer cases that men tend to be diagnosed at an older age than women and with a later stage of the disease [11]. Regarding the histological features, mucinous carcinomas are classified into pure and mixed types. The pure type shows $>90 \%$ of a mucinous component and is also classified into hypo cellular and hyper cellular types. It is associated with lower rates of recurrence and indolent behavior. The mixed type behaves as aggressively as the isolated, invasive, solid component $[12,13]$. The Authors present a mucinous mixed type of the male breast, which was initially diagnosed as a mucinous carcinoma of the skin, reporting its clinical and pathological features.

\section{Case Report}

In February 2017, a 69-year-old male presented at Sant'Andrea Hospital, Rome, with an enlarged lump in the right breast and a palpable lesion in his right axilla. In December 2011, the patient had noticed thickening of the skin in the armpit area. During the examination, ultrasonography (US) revealed a hypoechoic, dishomogeneous area in the right axilla of $2 \mathrm{~cm}$ in diameter. Doppler US showed that the mass had internal vascularity. An x-ray of the thorax was negative. US confirmed the presence of a palpable lesion of the axilla but did not show any lesions in the breast. Other clinical examinations and systemic investigations including chest, abdominal and pelvis computed tomographies, and thyroid, breast and genitourinary tract ultrasound failed to detect any primary lesion elsewhere. BRCA analysis was negative and no family history of carcinomas was reported. The patient underwent incisional surgery and histopathology diagnosed a mucinous carcinoma. The immunohistochemical evaluation, performed according to the standard procedure, showed that tumor cells were positive for cytokeratin 20 (Clone Ks20.8 Dako), weakly positive for CEA (Dako) and cytokeratin 7 (clone OV-TL 12/30, Dako) but negative for ER (clone EP1, Dako), PgR (clone PgR636, Dako), TTF1 (clone 8G7G1/1 Dako) and. Ki67 was

*Corresponding author: Ilaria Lamberti, Department of Clinical and Molecular Medicine, Sapienza University of Rome, Rome, Italy, Tel: +39-0633775345; E-mail: ilaria.lamberti@uniroma1.it

Received November 10, 2017; Accepted December 20, 2017; Published December 28, 2017

Citation: Lamberti I, Bonifacino A, Scarpino S, Villani S, Mancini R, et al. (2017) Clinical-Pathological Features of an Occult Mixed Mucinous Male Breast Cancer: A Case Report. J Cytol Histol 8: 493. doi: 10.4172/2157-7099.1000493

Copyright: @ 2017 Lamberti I, et al. This is an open-access article distributed under the terms of the Creative Commons Attribution License, which permits unrestricted use, distribution, and reproduction in any medium, provided the original author and source are credited. 
$30 \%$. On the basis of these results, the hypothesis of a cutaneous adnexal mucinous adenocarcinoma was suggested. In 2013, ultrasonography (US) revealed two hypoechoic solid masses in the right axillary cavity. Fine-needle-aspiration cytology (FNAC) confirmed the neoplasia in the right axillary cavity and a histopathological examination revealed a mucin-producing adenocarcinoma in all the lymph nodes examined. As a result, the patient received adjuvant radiation therapy for 1 month, followed by chemotherapy. In 2016, the clinical examination, US and PET/CT confirmed the presence of multiple metastatic lateral cervical, axillary and right mammary lymph node chains. An increased uptake was evident in the right mammary gland. The patient began consolidation chemotherapy with taxol from 02/2016 until 01/2017 with a good response (partial response). Other clinical examinations and systemic investigations, including breast ultrasound were requested.

In 2017 US revealed a hypoechoic solid mass with irregular margins in the retroareolar region of the right breast and two lymph nodes with irregular margins in the right axillary cavity (Figure 1). A FNAC, supported by Rapid On-Site Evaluation (ROSE), of the breast lesion was performed. The cytological evaluation showed, in all smears, the presence of blood and mucus in the background, higher cellularity sometimes with nuclear atypia, overlapping, crowding and three-dimensional clusters (Figure 2) supporting the hypothesis of a primary breast tumor. In the same session, a core biopsy was performed. Histopathology revealed infiltrating ductal structures characterized by evident nuclear atypia, anisonucleosis, prominent nucleoli and mitotic activity in an extensive mucinous background. The immunohistochemical pattern showed that ER and PgR were both negative, $\mathrm{p} 53$ positive (24\%), and c-erbB $2 /$ neu very positive (continuous and strong membrane reactivity in more than $50 \%$ of the cells) and the MIB-1 (clone MIB-1, Dako) clone was 30-40\%. Fluorescence in situ hybridization (FISH) assay for HER2 was amplified confirming the immunoreactivity (Figure 3). The lesion was also synaptophisin (Clone Synap, Dako) positive (Figure 4), confirming that the mucinous carcinoma is commonly associated with neurendocrine differentiation [4]. The histopathology diagnosis was a mixed mucinous and infiltrating carcinoma of the male breast. The patient received cycles of molecular target therapy and neoadjuvant chemotherapy. Then, in November 2017, the patient underwent a radical mastectomy.

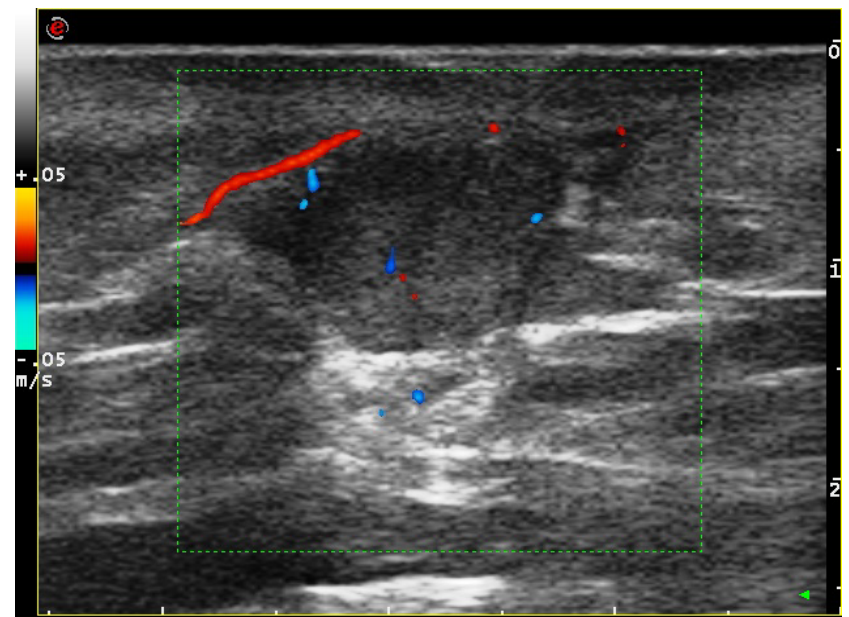

Figure 1: Right breast ultrasonography revealed a hypoechoic solid mass with irregular margins approximately $1 \mathrm{~cm}$ in maximum size in the retro areolar region.

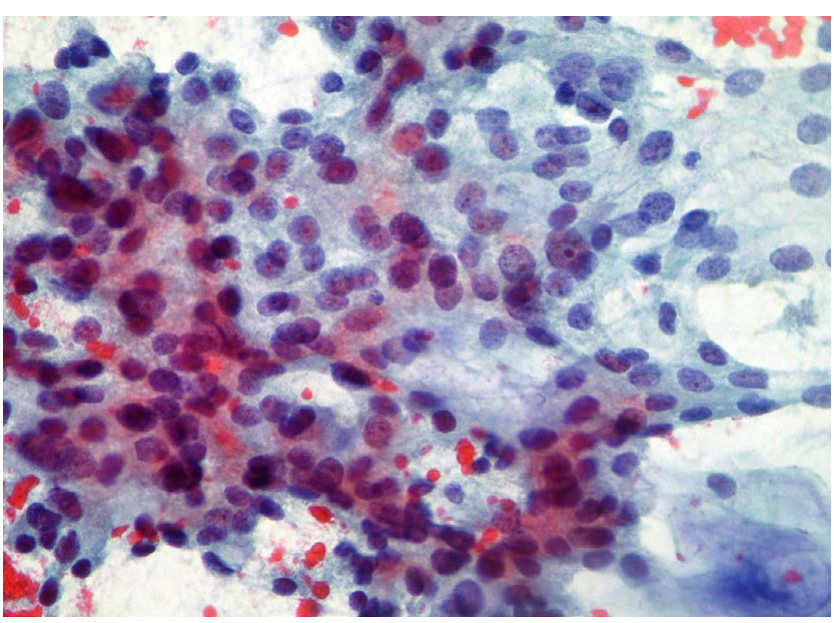

Figure 2: FNAC. Mucinous carcinoma showing pools of mucin and ductal cells. Nuclear atypia, overlapping and crowding is observe in cases of mixed mucinous and ductal carcinoma (Papanicolaou x200).

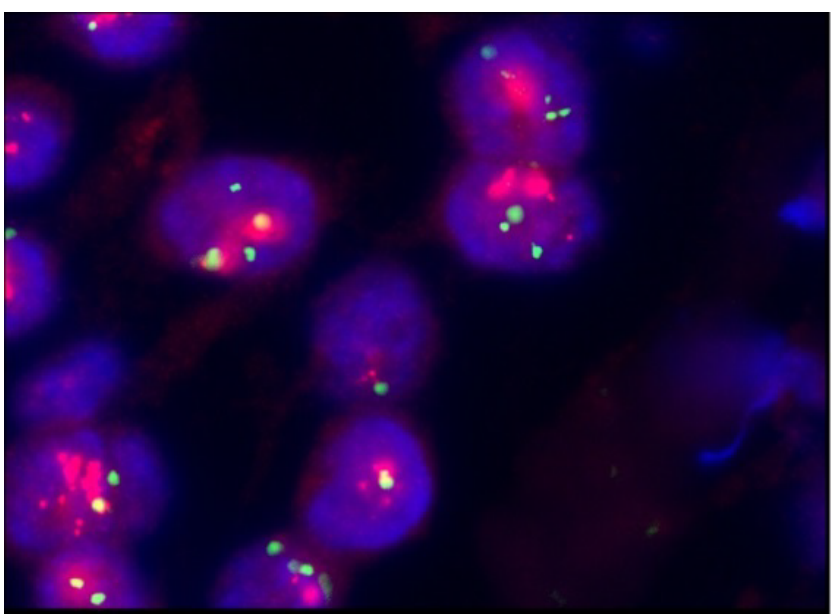

Figure 3: Fluorescence in situ hybridization (FISH) assay for HER2. This image shows the appearance of the dual-colored FISH assay (HER-2/neu DNA Probe Kit 17q 11.2-q12 Cromosoma 17 satellite alfa 17p11.1-q11.1, CEP17; Kreatech, Resnova, Italy), x1000 original magnification). Invasive tumor cell nuclei are highlighted by the blue fluorescent counter stain (4',6-diamidino-2- phenylindole [DAPI]). Figure show HER2-amplified breast cancer with an increased number of HER2 gene signals (red signals) relative to CEP17 (green signals) resulting in a calculated HER2/CE17 ration of greater than 2 .

\section{Discussion}

We presented a case of mucinous carcinoma initially diagnosed as a mucinous carcinoma of the skin. Seven years later, after excision at the primary site, it was classified as a mixed mucinous and infiltrating carcinoma of the breast. According to the literature, differential diagnosis between these tumors remains difficult. Three quarters of cases of mucinous carcinoma of the skin were found on the head while only $2 \%$ of mucinous carcinomas occurred on the chest [14]. Furthermore, mucinous carcinomas are classified into pure and mixed type, where the pure type shows the mucinous component in more than $90 \%$ of the tumor; on the contrary, the mixed type shows mucinous and solid components formed by a ductal in situ, intraductal (IDC) or neuroendocrine differentiation carcinoma. The pure mucinous type is further sub-classified into hypocellular (A) and hypercellular 


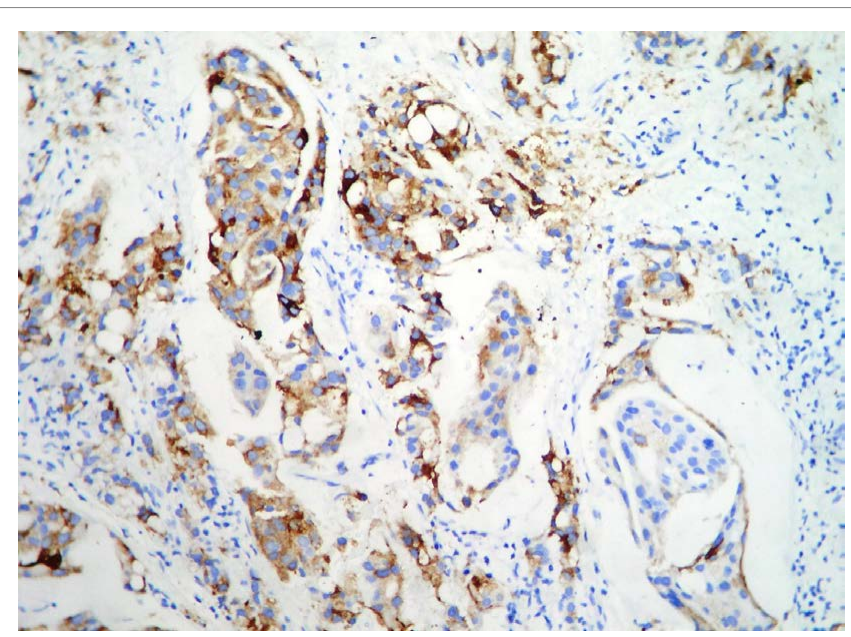

Figure 4: Mucinous breast cancer shows IHC expression of synaptophisin in the vast majority of cancer cells.

(B) types with a lower rate of recurrence and an indolent behavior; on the contrary, the mixed type proves to have more aggressive behavior $[12,13,15]$. At the first step of clinical investigation, our case presented a hypoechoic dishomogeneous area in the right axilla discovered by US. The lesion was treated by surgical excision and the histopathological evaluation showed a mixed type of mucinous carcinoma involving the skin. Clinical examinations and systemic investigations including chest, abdominal and pelvis computed tomographies, and thyroid, breast and genitourinary tract ultrasound failed to detect any primary lesion elsewhere. Analysis of various aspects of mammary carcinomas in men suggests some reasons for their worse behavior. Carcinomas of the male breast are usually superficial and beneath the nipple where the majority of mammary ducts are present. It is known that cancers in central locations of the breast in women have a worse prognosis than those in the more favorable areas such as the outer half of the breast. Also, the male breast lacks sufficient substance to encompass the tumor, so that the neoplasm has ready access to dermal lymphatics, pectoral fascia and subareolar lymphatic channels, metastatizing earlier in the axillary lymph nodes [16]. MBC with an unknown primary lesion, also defined as occult breast cancer, is a rare event and presents a "special kind" of breast cancer, of which the initial symptom is axillary lymphatic metastasis. Occult breast cancer is a type of cancer in which no lesions are found through physical or radiological examination such as mammography. It does not usually show any symptoms in the breast and it is often discovered from lymph node metastasis in the axillary area, supraclavicular or infraclavicular fossa [17]. In the case where only metastatic lesions are detected, without an original lesion, this is defined as carcinoma of unknown primary. In our case the first clinical manifestation was a skin mass of the armpit area. Even when the metastasis is detected in an axillary lymph node, it is difficult to be sure that occult is breast cancer. Although the axillary lymph node is known to be highly relevant to breast cancer, many other malignancies can metastatize to axillary lymph nodes, including lymphoma, melanoma, lung, thyroid and digestive tract carcinomas [18]. In the male patient studied, no breast mass was found at the first physical examination of the patient, not even a primary tumor was observed at US and PET/CT tests. After surgical removal of the skin lesion, the immunohistochemical tests showed that tumor cells were negative for ER and PgR. The literature reports that male breast cancers have high rates of hormone-receptor expression. Giordano et al detected that approximately $90 \%$ of male breast cancers express the estrogen receptor, and $81 \%$ express the progesterone receptor [11]. In contrast, the her2-neu proto-oncogene is less likely to be overexpressed in cancers of the male breast. In a large series of studies, human epidermal growth factor receptor-2 (HER-2) was observed in only one tumor out of 58 male breast cancers showing overexpression without amplification, compared with $26 \%$ of female breast cancer tumors showing overexpression and $27 \%$ manifesting amplification [3]. At present, there are no reports in the literature of MBC cases showing a mucinous carcinoma which is negative for $\mathrm{ER} / \mathrm{PgR}$ and Her2neu amplified. This pattern is extremely uncommon in MBC. In fact, the literature reports that the immunoprofiles of mucinous carcinoma in males and females show fundamentally the same patterns $[7,12]$. Nevertheless, $\mathrm{Ha}$ et al reported a case of invasive mucinous breast carcinoma in a woman in which the lesion was estrogen and progesterone receptor negative and HER-2 positive [19]. Studies have revealed that there is a subset of patients with mucinous carcinoma showing mammographically occult neoplasms and/or multicentric multifocal disease. Some of these patients demonstrated metastases at presentation as well as ER/PR negative and HER-2 positive [20,21].

\section{Conclusion}

In these cases, immunohistochemistry was misleading. The presence of both negative hormonal receptors gave information that confused the diagnosis. Moreover, the histological pattern of a colloid carcinoma supported the hypothesis of a rare skin mucinous adenocarcinoma of the axilla, by ruling out other primary cancers. In conclusion, from our experience, in the presence of a lesion in the axillary area with no specific primary origin, breast cancer should never be ruled out, even in the absence of clinical evidence and with an immunohistochemical pattern not indicative of mammary origin. In this unusual case, it is probable that FNAC contributed significantly to the diagnosis and that the collaboration between the clinician and the pathologist led to a correct evaluation. The presence of the classic pattern of breast colloid carcinoma, found by breast FNAC, made the diagnosis evident. In this case, the cytopathology evaluations suggest a new hypothesis on the primary origin of the lesion, which was later supported by the histological examination.

\section{References}

1. Ouyang XN (2014) Misdiagnosed male breast cancer with an unknown primary tumor: A case report. Oncol Lett 8: 190-192.

2. O'Malley CD, Prehn AW, Shema SJ, Glaser SL (2002) Racial/ethnic differences in survival rates in a population-based series of men with breast carcinoma. Cancer 94: 2836-2843.

3. Fentiman IS, Fourquet A, Hortobagyi GN (2006) Male breast cancer. Lancet 367: 595-604.

4. Ishida M, Umeda T, Kawai $Y$, Mori T, Kubota $Y$, et al. (2014) Mucinous carcinoma occurring in the male breast. Oncol Lett 7: 378-380.

5. Dawson PJ, Paine TM, Wolman SR (1992) Immunocytochemical characterization of male breast cancer. Mod Pathol 5: 621-625.

6. Fox SB, Day CA, Rogers S (1991) Lack of c-erbB-2 oncoprotein expression in male breast carcinoma. J Clin Pathol 44: 960-961.

7. Muir D, Kanthan R, Kanthan SC (2003) Male versus female breast cancers. A population-based comparative immunohistochemical analysis. Arch Pathol Lab Med 127: 36-41.

8. Hittmair AP, Lininger RA, Tavassoli FA (1998) Ductal carcinoma in situ (DCIS) in the male breast: a morphologic study of 84 cases of pure DCIS and 30 cases of DCIS associated with invasive carcinoma a preliminary report. Cancer 83: 2139-2149.

9. Javidiparsijani S, Rosen LE, Gattuso P (2017) Male Breast Carcinoma: A 
Citation: Lamberti I, Bonifacino A, Scarpino S, Villani S, Mancini R, et al. (2017) Clinical-Pathological Features of an Occult Mixed Mucinous Male Breast Cancer: A Case Report. J Cytol Histol 8: 493. doi: 10.4172/2157-7099.1000493

Clinical and Pathological Review. Int J Surg Pathol 25: 200-205.

10. Burga AM, Fadare O, Lininger RA, Tavassoli FA (2006) Invasive carcinomas of the male breast: a morphologic study of the distribution of histologic subtypes and metastatic patterns in 778 cases. Virchows Arch 449: 507-512.

11. Giordano SH (2005) A review of the diagnosis and management of male breast cancer. Oncologist 10: 471-479.

12. Kazakov DV, Suster S, LeBoit PE, Calonje E, Bisceglia M, et al. (2005) Mucinous carcinoma of the skin, primary, and secondary: a clinicopathologic study of 63 cases with emphasis on the morphologic spectrum of primary cutaneous forms: homologies with mucinous lesions in the breast. Am J Surg Pathol 29: 764-782.

13. Bae SY, Choi MY, Cho DH, Lee JE, Nam SJ, et al. (2011) Mucinous carcinoma of the breast in comparison with invasive ductal carcinoma: clinico pathologic characteristics and prognosis. J Breast Cancer 14: 308-313.

14. Snow SN, Reizner GT (1992) Mucinous eccrine carcinoma of the eyelid. Cancer 70: 2099-2104.

15. S Kashiwagi, N Onoda, Y Asano, H Kawajiri, T Takashima, M Ohsawa, et al.
(2013) Clinical significance of the sub-classification of 71 cases mucinous breast carcinoma. Springer Plus 2: 481-486.

16. Norris HJ, Taylor HB (1969) Carcinoma of the male breast. Cancer 23: 1428 1435.

17. Baron PL, Moore MP, Kinne DW, Candela FC, Osborne MP, et al. (1990) Occult breast cancer presenting with axillary metastases. Updated management. Arch Surg.125: 210-214.

18. Copeland EM, McBride CM (1973) Axillary metastases from unknown primary sites. Ann Surg 178: 25-27.

19. Ha KY, Deleon P, Deleon W (2013) Invasive mucinous carcinoma of the breast Proc (Bayl Univ Med Cent) 26: 295-297.

20. Shet T, Chinoy R (2008) Presence of a micro papillary pattern in mucinous carcinomas of the breast and its impact on the clinical behavior. Breast $\mathrm{J} 14$ 412-420.

21. Perkins G, Babiera G, Bedrosian I, Gonzalez-Angulo A, Whitman G, et al (2009) Mucinous breast carcinoma: occult multifocality/multicentricity in a favorable disease. Cancer Res 69: 4117. 